\title{
EFEITO DA ADIÇÃO DE ELEMENTOS DE LIGA NA ABSORÇÃO DE HIDROGÊNIO EM LIGAS A BASE DE ZIRCÔNIO-NIÓBIO PARA APLICAÇÃO NUCLEAR*
}

\section{Resumo}

Rodrigo Vitorino da Silva ${ }^{1}$ Rafaella Martins Ribeiro² Dilson Silva dos Santos ${ }^{3}$

Ligas a base de zircônio utilizadas na indústria nuclear estão sujeitas à formação de hidretos que são causadores de efeito deletério no material. Para aumentar o tempo de operação desses componentes, é necessário o desenvolvimento de novas ligas que retardem ou diminuam esse efeito. Este trabalho tem como objetivo avaliar a influência da adição de elementos de liga em uma liga inédita a base de $\mathrm{Zr}-\mathrm{Nb}$ na absorção de hidrogênio, através de testes que avaliam a absorção de hidrogênio e a formação de hidretos no material após processamento termomecânico e comparar seu desempenho com duas ligas comerciais. Para tal, foram realizados ensaios de microdureza, cinética de absorção de hidrogênio e micrografias. Os resultados de microdureza Vickers das três ligas estudadas, após o processamento termomecânico, são muito próximos. As curvas de cinética de absorção de hidrogênio mostram que a liga Zr-Nb-Sn-Fe apresenta melhor comportamento que as demais uma vez que o tempo para o início de absorção foi maior. As micrografias indicam que a liga proposta apresenta um desempenho superior em relação à morfologia dos hidretos visto que os mesmos são mais curtos e alinhados.

Palavras-chave: Nuclear; Liga de zircônio; Hidrogênio; Hidreto.

\section{THE EFFECT OF ALLOY ELEMENTS ADDITION IN THE HYDROGEN ABSORPTION ON ZIRCONIUM-NIOBIUM BASED ALLOY FOR NUCLEAR APPLICATION}

\section{Abstract}

Zirconium based alloys used in nuclear industry are subject the formation of hydrides which have a deleterious effect in the material. To increase the operating time of these components is necessary to develop new alloys that delay or reduce this effect. The aim of this work is to evaluate the influence of alloying elements addition in a new $\mathrm{Zr}-\mathrm{Nb}$-based alloy in hydrogen absorption through tests that evaluate the hydrogen absorption and the formation of hydrides in the material, which underwent thermomechanical process, and to compare your performance with two commercial alloys. For this purpose, microhardness test, hydrogen absorption kinetics test and SEM micrographs were done. The Vickers microhardness test reports, after the thermomechanical treatment, the three alloys present similar hardness values. The kinetics hydrogen absorption shows that the $\mathrm{Zr}-\mathrm{Nb}-\mathrm{Zn}-\mathrm{Fe}$ alloy presents a better behavior in comparison with the others once it took longer to start the hydrogen absorption process. The SEM micrographs indicate that the proposed alloy presents a superior performance among the analyzed samples due to the shorter and aligned morphology of the hydrides.

Keywords: Nuclear; Zirconium alloy; Hydrogen; Hydrides.

1 Graduando em Engenharia Metalúrgica do PEMM - COPPE / Universidade Federal do Rio de Janeiro, RJ, Brasil.

2 Doutora em Eng. Metalúrgica e Materiais e Professora do PEMM - COPPE / Universidade Federal do Rio de Janeiro, RJ, Brasil.

3 Físico, DSc em Eng. Metalúrgica e Materiais e Professor do PEMM - COPPE / Universidade Federal do Rio de Janeiro, RJ, Brasil. 


\section{INTRODUÇÃO}

As ligas a base de zircônio são utilizadas para a fabricação das varetas combustíveis de reatores nucleares devido às suas propriedades de baixa absorção de nêutrons e alta resistência à corrosão em ambientes de alta temperatura e pressão [1].

Nas condições de operação dos reatores PWR brasileiros $\left(320^{\circ} \mathrm{C}, 160 \mathrm{~atm}\right)$ ocorre a decomposição radiolítica da água e o hidrogênio é absorvido, podendo levar à formação de hidretos quando o hidrogênio absorvido excede o limite de solubilidade na liga, reduzindo a ductilidade e a tenacidade à fratura. A orientação dos hidretos depende da textura desenvolvida durante a fabricação. Hidretos na direção circunferencial são aqueles paralelos à superfície da vareta e hidretos na direção radial são aqueles perpendiculares à superfície da vareta. Hidretos radiais limitam a vida em serviço da vareta combustível e reduzem a queima de combustível nos reatores. A melhor maneira de retardar ou reduzir a absorção de hidrogênio é através do controle dos processos de fabricação das varetas.

Este trabalho tem como objetivo avaliar a influência da adição de elementos de liga em ligas a base de $\mathrm{Zr}-\mathrm{Nb}$ na absorção de hidrogênio. Foram estudadas três ligas, duas comerciais e uma de composição inédita que está sendo desenvolvida para futura aplicação nos reatores brasileiros. A avaliação de seus desempenhos se deu através da fusão das ligas, processamento termomecânico e tratamento térmico para adequação da microestrutura. As três ligas foram submetidas ao mesmo processamento. $O$ efeito do hidrogênio e as condições de formação do hidreto nas ligas foram determinados através de ensaios de cinética de absorção de hidrogênio e microscopia ótica. Os resultados permitiram comparar o desempenho da liga proposta com as ligas comerciais.

\section{MATERIAIS E MÉTODOS}

\subsection{Materiais}

Para o desenvolvimento deste trabalho foram utilizadas três ligas de zircônio: duas comerciais, $\mathrm{Zr}-1 \mathrm{Nb}$ e $\mathrm{Zr}-1 \mathrm{Nb}-1 \mathrm{Sn}-0,1 \mathrm{Fe}$; e uma de composição inédita, cuja composição é $\mathrm{Zr}-0,8 \mathrm{Nb}-0,2 \mathrm{X}-0,5 \mathrm{Y}$ onde $\mathrm{X}$ é um metal de transição e $\mathrm{Y}$ é um metal representativo. Utilizaremos ao longo do texto a nomenclatura $\mathrm{Zr}-\mathrm{Nb}, \mathrm{Zr}-\mathrm{Nb}-\mathrm{Sn}-\mathrm{Fe}$ e Zir-Brasil-2 para diferenciar estas três ligas. Os níveis de pureza dos elementos utilizados na produção das ligas são apresentados na tabela 1.

Tabela 1. Pureza dos elementos utilizados na produção das ligas

\begin{tabular}{cc}
\hline Elemento & Pureza (\%) \\
\hline $\mathrm{Zr}$ & 99,99 \\
\hline $\mathrm{Nb}$ & 99,70 \\
\hline $\mathrm{Sn}$ & 99,95 \\
\hline $\mathrm{Fe}$ & 99,98 \\
\hline $\mathrm{X}$ & 99,95 \\
\hline $\mathrm{Y}$ & 99,80 \\
\hline
\end{tabular}




\subsubsection{Fusão das ligas}

A fusão das três ligas foi realizada no forno elétrico a arco Bühler AM VARIO 400. As cargas foram fundidas sob uma pressão de 200 mbar de gás argônio de alta pureza e corrente entre 120 e $150 \mathrm{~A}$, resultando em lingotes de $60 \mathrm{~g}$ com $9 \mathrm{~mm}$ de espessura e $100 \mathrm{~mm}$ de comprimento, aproximadamente.

\subsection{Processamento termomecânico}

\subsubsection{Laminação a quente}

A laminação a quente foi realizada à temperatura de $850^{\circ} \mathrm{C}$ no laminador FENN 150 - HARTFORD, CONN, HORSBURG e SCOTT. Os lingotes das três ligas foram aquecidos em um forno Carbolite por 15 minutos e submetidos a 3 passes de laminação de $1,5 \mathrm{~mm}$. Devido à perda de calor, eles retornaram ao forno com objetivo de recuperarem a temperatura e sofrerem novos 3 passes de laminação, onde obteve-se um grau de redução de $83 \%$ e espessura final de $1,5 \mathrm{~mm}$.

\subsubsection{Laminação a frio}

As três chapas oriundas da laminação a quente foram laminadas a frio no laminador FENN 150 - HARTFORD, CONN, HORSBURGH e SCOTT à temperatura ambiente, onde obteve-se um grau de redução de $40 \%$ através de 6 passes de 0,2 $\mathrm{mm}$. Nesta etapa foram obtidas três chapas com $0,9 \mathrm{~mm}$ de espessura.

\subsubsection{Tratamento térmico}

Foi realizado nas chapas das três ligas um tratamento térmico de $600^{\circ} \mathrm{C}$ por $2 \mathrm{~h}$ em um forno tubular Carbolite, com resfriamento ao ar. As amostras foram encapsuladas em tubos de quartzo sob vácuo para reduzir a oxidação superficial durante o tratamento térmico.

\subsection{Ensaio de microdureza}

As durezas Vickers das ligas após tratamento térmico foram obtidas utilizando um microdurômetro Zwick/Roell Indentec Zhu-Mkll-M. Os parâmetros utilizados para a realização das medidas foram: carga de $200 \mathrm{~g}$ e tempo de $15 \mathrm{~s}$.

\subsection{Cinética de absorção de hidrogênio}

As medidas de cinética de absorção de hidrogênio foram realizadas no equipamento PCT Pro 2000 HyEnergy, numa temperatura de $320^{\circ} \mathrm{C}$ sob uma pressão de 10 bar de $\mathrm{H}_{2}$ para determinar o percentual em peso de hidrogênio absorvido e o tempo em que a absorção ocorre. Estas condições foram escolhidas para simular as condições de operação do reator nuclear.

\subsection{Hidrogenação por carregamento gasoso}

Para induzir a formação de hidretos, as amostras das três ligas foram colocadas em uma autoclave sob uma pressão de 10 bar de $\mathrm{H}_{2}$. A autoclave foi colocada em um forno Carbolite numa temperatura de $320^{\circ} \mathrm{C}$ por $48 \mathrm{~h}$. 


\subsection{Microscopia ótica}

A microscopia ótica permitiu observar a orientação e a distribuição dos hidretos após a hidrogenação. O microscópio utilizado para a obtenção das imagens foi o Olympus BX60MF com aumento de 50x e100x.

\section{RESULTADOS E DISCUSSÃO}

\subsection{Fusão das ligas}

As fusões das ligas $\mathrm{Zr}$-Nb e $\mathrm{Zr}$-Nb-Sn-Fe ocorreram em uma única etapa na qual todos os elementos foram colocados juntos e fundidos em uma única vez. Entretanto, foi necessário realizar co-fusões na Zir-Brasil-2 para garantir a dissolução dos elementos de liga no $\mathrm{Zr}$ e a inexistência de segregações. As três ligas foram fundidas e refundidas cinco vezes para garantir a homogeneização da composição no lingote. Em cada refusão o lingote foi girado $180^{\circ}$ para verificação do aspecto superficial e favorecer a homogeneização. A figura 1 mostra o aspecto final do lingote da liga Zir-Brasil-2.

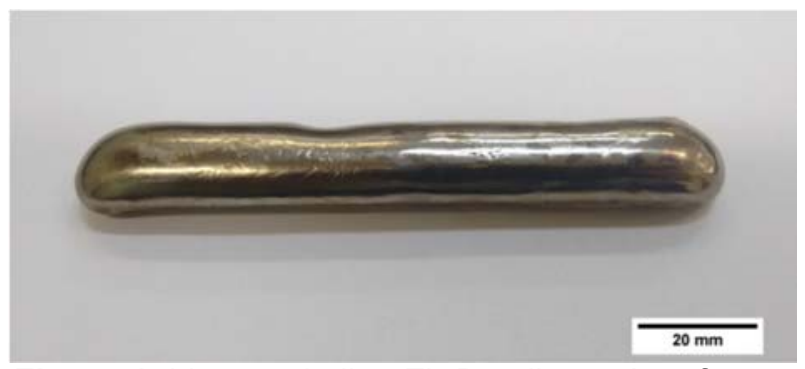

Figura 1. Lingote da liga Zir-Brasil-2 após a fusão.

\subsection{Laminação a quente e a frio}

Ao final das laminações, as chapas apresentaram o mesmo comportamento com pequenas trincas ao longo do seu comprimento devido ao esforço que elas sofreram durante a laminação a frio. A figura 2 mostra o aspecto final da chapa da liga ZirBrasil-2.

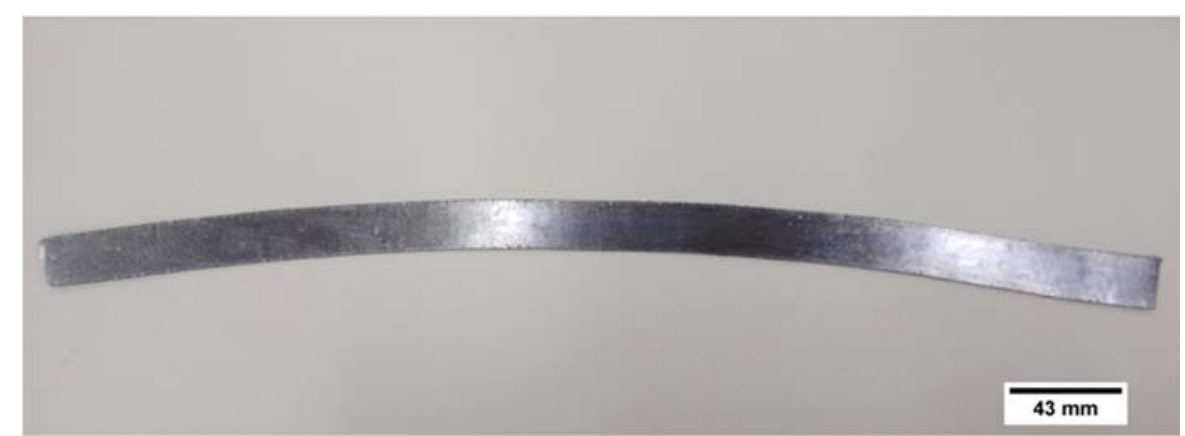

Figura 2. Chapa da liga Zir-Brasil-2 após a laminação.

\subsection{Tratamento térmico}

Para a determinação do tempo e temperatura que seriam utilizados no tratamento térmico, foi realizado o ensaio de microdureza Vickers. A liga utilizada foi a que está sendo desenvolvida no laboratório, de composição inédita, Zir-Brasil-2. O objetivo 
era realizar um tratamento térmico onde obtivesse uma microestrutura recristalizada, com grãos pequenos e precipitados finamente dispersos na matriz para que os hidretos formados após a hidrogenação assumissem uma direção preferencial. A tabela 2 mostra os valores de dureza Vickers obtidos para diferentes condições.

Tabela 2. Dureza Vickers da liga Zir-Brasil-2 em diferentes condições.

\begin{tabular}{cc}
\hline Condição & $\begin{array}{c}\text { Dureza } \\
\text { Vickers (HV) }\end{array}$ \\
\hline Fundida & 221 \\
\hline Laminada & 271 \\
\hline $600^{\circ} \mathrm{C} 1 \mathrm{~h}$ & 251 \\
\hline $600^{\circ} \mathrm{C} 2 \mathrm{~h}$ & 241 \\
\hline $600^{\circ} \mathrm{C} 6 \mathrm{~h}$ & 215 \\
\hline $600^{\circ} \mathrm{C} 12 \mathrm{~h}$ & 220 \\
\hline
\end{tabular}

Os resultados mostram uma redução do valor de dureza até o tratamento de $600^{\circ} \mathrm{C}$ por $6 \mathrm{~h}$, este comportamento foi encontrado em [2] ao estudar a liga Zr-Nb em condições similares. Para o tratamento térmico de $600^{\circ} \mathrm{C}$ por $12 \mathrm{~h}$, a dureza subiu, indicando que houve uma precipitação. O decaimento da dureza nos tratamentos térmicos a $600^{\circ} \mathrm{C}$ por 1,2 e 6 horas mostra que o grau de recristalização foi aumentando de acordo com o tempo de tratamento a ponto do baixo valor da dureza encontrado no tratamento térmico de $600^{\circ} \mathrm{C}$ por 6 horas indicar que a amostra estava totalmente recristalizada, com grãos maiores do que os objetivados.

Com base nos resultados obtidos no ensaio de microdureza, foi definido que o tratamento térmico realizado seria a uma temperatura de $600^{\circ} \mathrm{C}$ por 2 horas.

\subsection{Ensaio de microdureza}

Os valores da dureza Vickers das três ligas em estudo após o tratamento térmico de $600^{\circ} \mathrm{C}$ por 2 horas é mostrado na tabela 3.

Tabela 3. Dureza Vickers das ligas em estudo após o tratamento térmico.

\begin{tabular}{cc}
\hline Liga & $\begin{array}{c}\text { Dureza } \\
\text { Vickers (HV) }\end{array}$ \\
\hline Zir-Brasil-2 & 241 \\
\hline Zr-Nb & 238 \\
\hline Zr-Nb-Sn-Fe & 240 \\
\hline
\end{tabular}

Os resultados mostram que, para o tratamento térmico proposto, as durezas Vickers das três ligas estão bem próximas, indicando que o tratamento escolhido permite obter propriedades mecânicas similares entre as ligas.

\subsection{Cinética de absorção de hidrogênio}

A figura 3 mostra que todas as ligas absorveram aproximadamente a mesma quantidade de hidrogênio em tempos diferentes. 


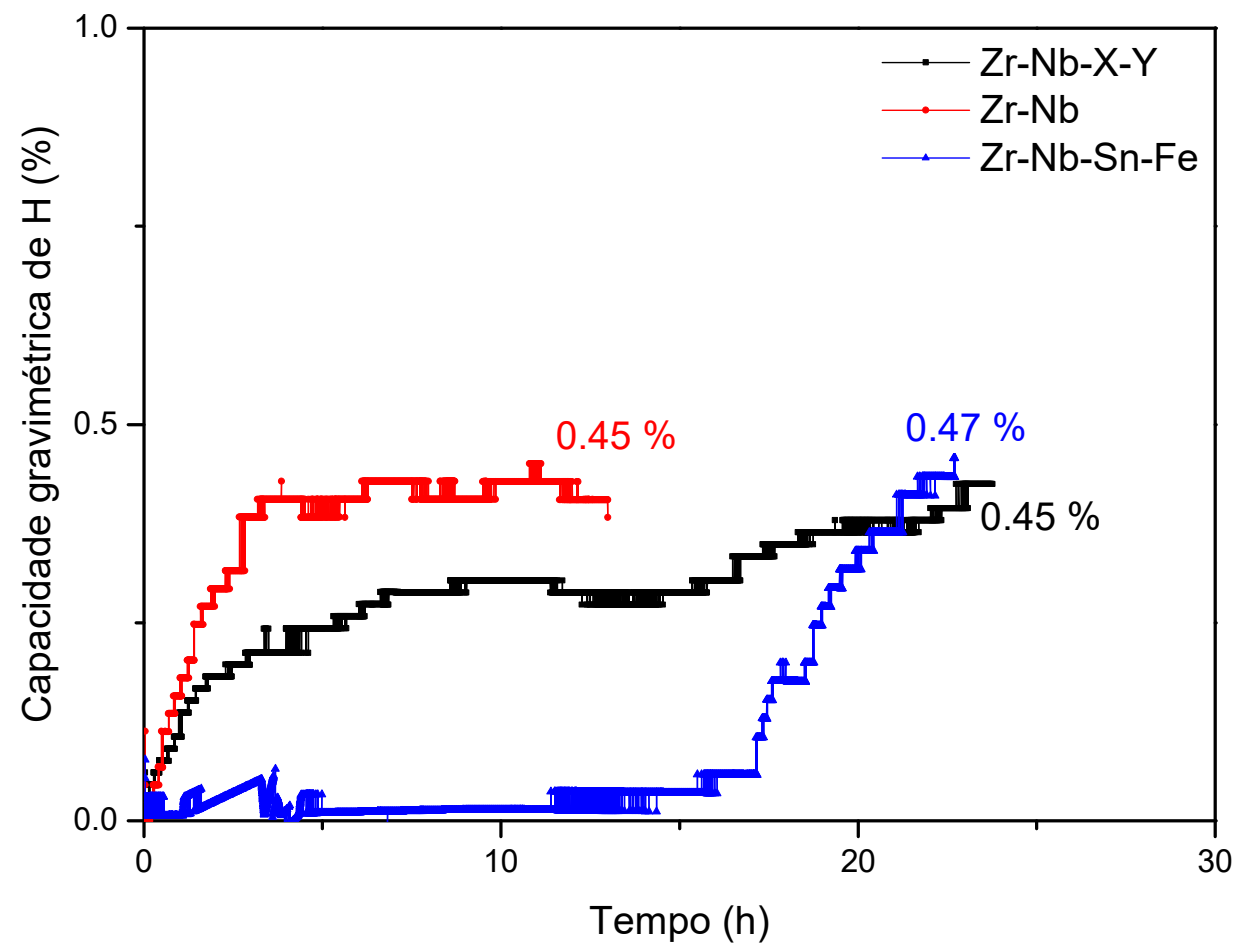

Figura 3. Curvas de cinética de absorção de hidrogênio das ligas a $320^{\circ} \mathrm{C}$ sob 10 bar de $\mathrm{H}_{2}$.

A liga Zr-Nb apresentou uma absorção inicial assim que começou a ser submetida às condições de hidrogenação, chegando à capacidade máxima absorvida em 11 horas. A liga Zir-Brasil-2 também apresentou uma absorção inicial, porém em menor quantidade se comparada à liga $\mathrm{Zr}-\mathrm{Nb}$, atingindo um estado estacionário quando chegou a 0,29 \%H absorvido. Após 16 horas de ensaio, a liga Zir-Brasil-2 voltou a absorver hidrogênio, chegando à mesma quantidade absorvida que a Zr-Nb em 23 horas. A liga Zr-Nb-Sn-Fe não apresentou uma absorção inicial, permanecendo sem absorver hidrogênio por 12 horas e atingiu uma quantidade absorvida de 0,47 \%H após 23 horas.

As curvas de cinética mostram que a liga Zr-Nb-Sn-Fe apresenta melhor comportamento que as demais uma vez que o tempo para o início de absorção foi maior. Ao estudar ligas de $\mathrm{Zr}-\mathrm{Nb}$ com a adição de $\mathrm{Al}, \mathrm{Cu}$ e $\mathrm{Mn}$ em diferentes condições de processamento termomecânico, Ribeiro [3] observou que em todas as condições analisadas as ligas absorveram pelo menos $0,59 \% \mathrm{H}$. Outros ensaios serão realizados para confirmar estes comportamentos.

\subsection{Microscopia ótica}

As figuras 4 a 6 mostram as imagens de microscopia ótica da seção transversal das três ligas tratadas termicamente após a hidrogenação por via gasosa a $320^{\circ} \mathrm{C}$ sob pressão de 10 bar por 48 horas. 

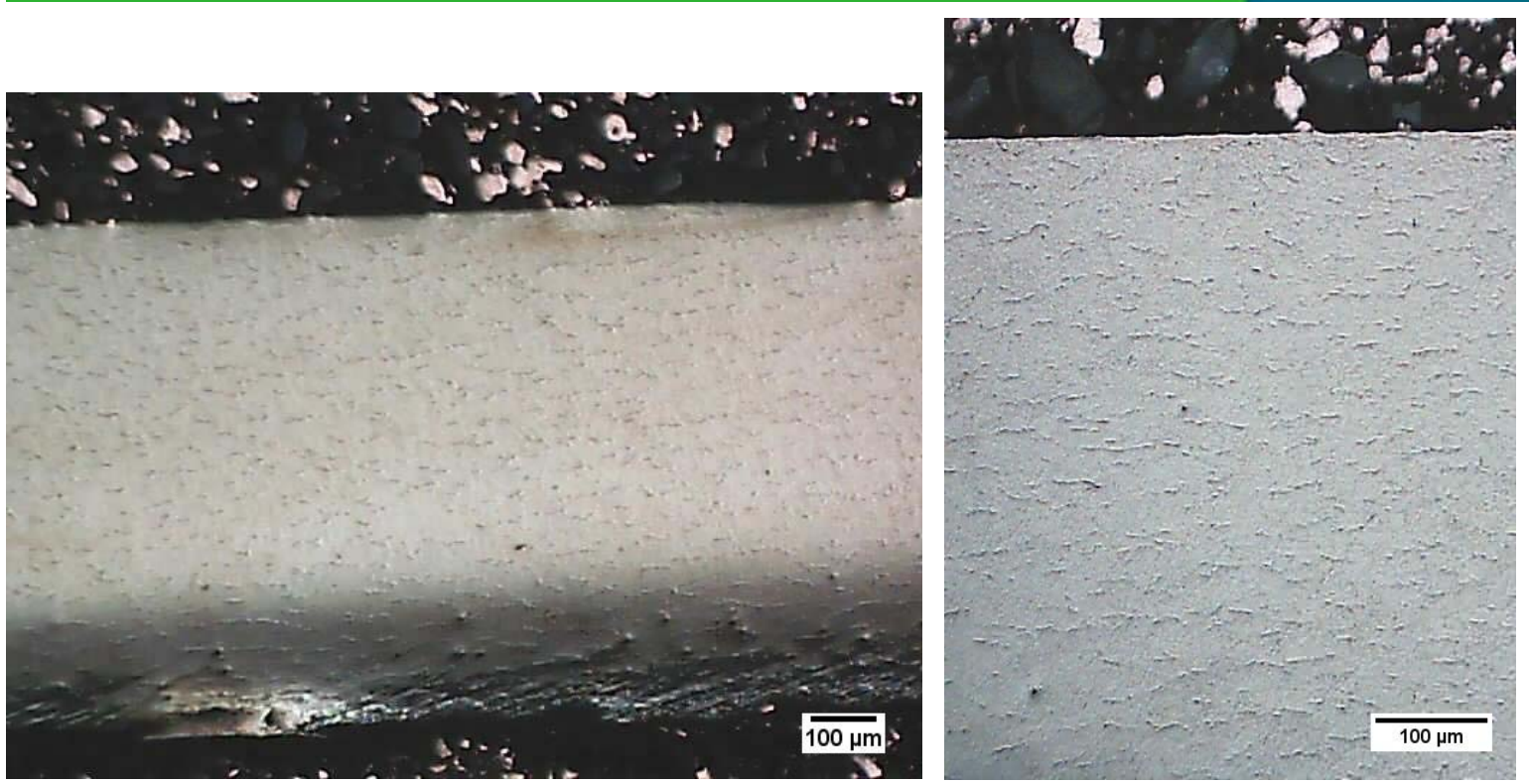

Figura 4. Micrografias da liga Zr-Nb-Fe-Sn tratada termicamente após hidrogenação.
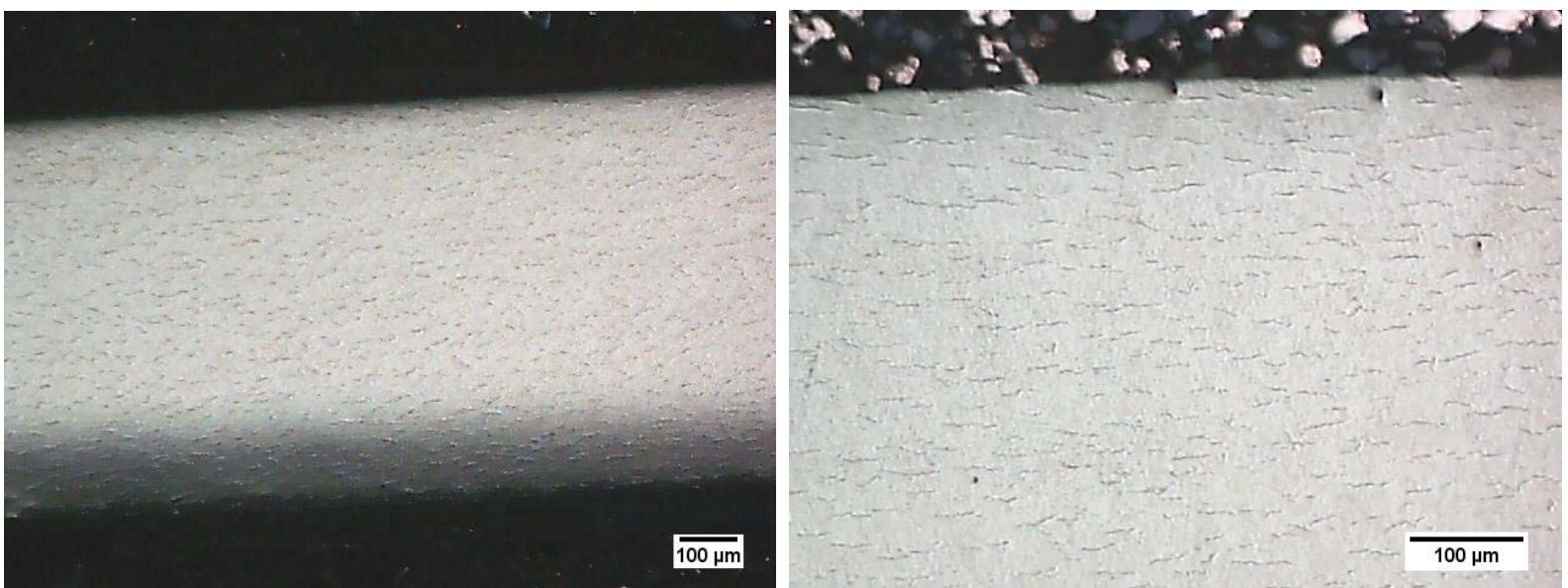

Figura 5. Micrografias da liga Zr-Nb tratada termicamente após hidrogenação.
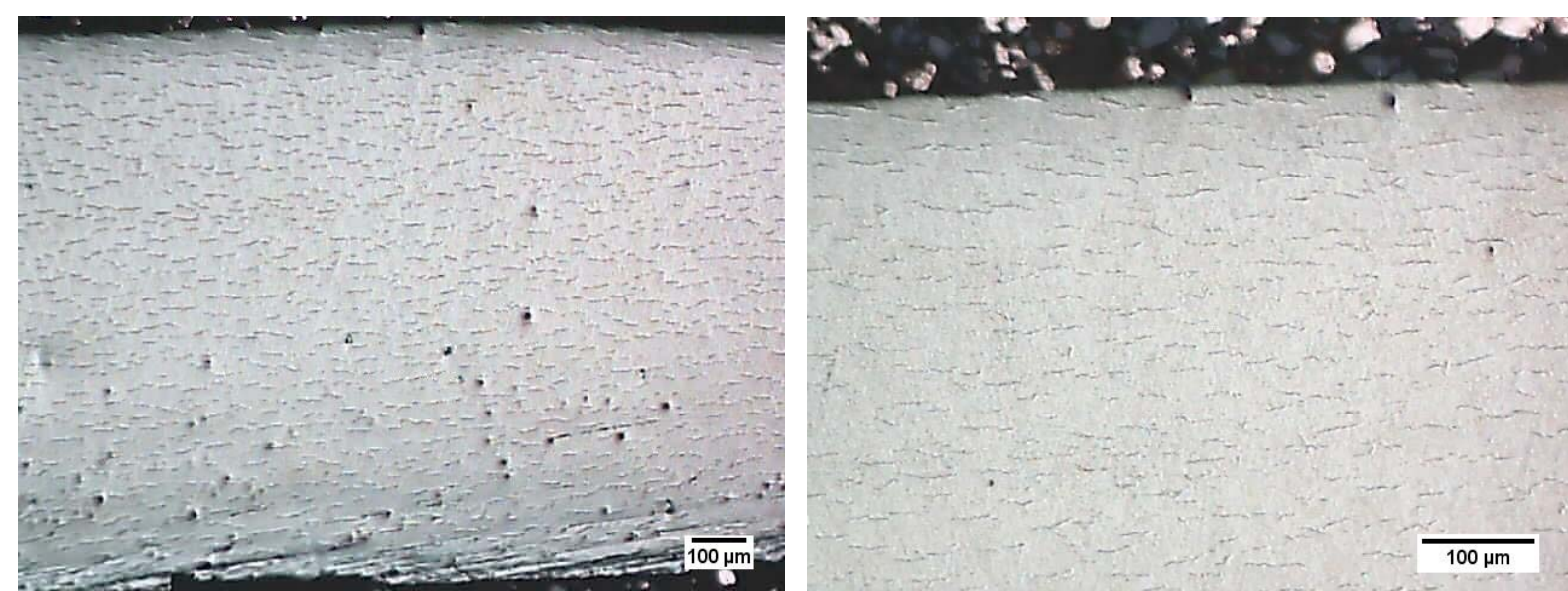

Figura 6. Micrografias da liga Zir-Brasil-2 tratada termicamente após hidrogenação.

As micrografias mostram uma distribuição uniforme de hidretos ao longo de toda espessura das amostras, seguindo uma direção transversal à direção da laminação, similar aos hidretos circunferenciais encontrados nas varetas combustíveis [4]. Este alinhamento dos hidretos ao plano basal ocorre devido à textura final gerada durante o processamento termomecânico das ligas. 
Os hidretos presentes na liga $\mathrm{Zr}-\mathrm{Nb}-\mathrm{Sn}-\mathrm{Fe}$ estão mais alongados e em menor quantidade se comparado às outras ligas. Os hidretos presentes na liga Zir-Brasil-2 estão mais alinhados, porém em maior quantidade se comparado à liga $\mathrm{Zr}-\mathrm{Nb}$. A mesma configuração de hidretos foi observada por Silva [5] em ligas $\mathrm{Zr}-\mathrm{Nb}$ e $\mathrm{Zr}-\mathrm{Nb}-$ $\mathrm{Sn}-\mathrm{Fe}$ nas mesmas condições. Os resultados obtidos indicam que a liga Zir-Brasil-2 apresenta um desempenho superior em relação à morfologia dos hidretos visto que os mesmos são mais curtos e alinhados.

\section{CONCLUSÃO}

Os resultados de ensaio de microdureza mostram que os valores de dureza Vickers das três ligas estudadas após o tratamento térmico são muito próximos, aproximadamente $240 \mathrm{HV}$.

As curvas de cinética de absorção de hidrogênio mostram que a liga $\mathrm{Zr}-\mathrm{Nb}$-Sn-Fe apresenta melhor comportamento que as demais uma vez que o tempo para o início de absorção foi maior para uma mesma quantidade absorvida, independente da composição estudada.

As micrografias indicam que a liga Zir-Brasil-2 apresenta um desempenho superior em relação à morfologia dos hidretos visto que os mesmos são mais curtos e alinhados.

\section{REFERÊNCIAS}

1 Vicente Alvarez MA, Santisteban JR, Vizcaino P, Flores AV, Banchik AD, Almer J. Hydride reorientation in $\mathrm{Zr} 2.5 \mathrm{Nb}$ studied by synchrotron $\mathrm{X}$-ray diffraction. Acta Materialia. 2012; 60:6892-6906.

2 Tian $\mathrm{H}$, Wang X, Gong W, Zhou J, Zhang H. Recrystallization behavior of cold-rolled Zr$1 \mathrm{Nb}$ alloy. Journal of Nuclear Materials. 2014; 456(2015):321-328.

3 Ribeiro RM. Interação hidrogênio-microestrutura em ligas a base de $\mathrm{Zr}$-Nb para aplicação em reatores nucleares. Tese de D.Sc., COPPE/UFRJ, Rio de Janeiro, RJ, Brasil. 2014.

4 Gabriel CW. Estudo da precipitação de hidretos em ligas de zircônio para aplicações nucleares. Dissertação de M.Sc., COPPE/UFRJ, Rio de Janeiro, RJ, Brasil. 2011.

5 Silva KFR. Interação do hidrogênio com a microestrutura de ligas de zircônio usadas em reatores nucleares: Estudo das propriedades mecânicas e difusão. Dissertação de M.Sc., COPPE/UFRJ, Rio de Janeiro, RJ, Brasil. 2009. 